\title{
Sirolimus therapy for fetal cardiac rhabdomyoma in a pregnant woman with tuberous sclerosis
}

\author{
Hyea Park, Chi Son Chang, Suk-Joo Choi, Soo-young Oh, Cheong-Rae Roh \\ Department of Obstetrics and Gynecology, Samsung Medical Center, Sungkyunkwan University School of Medicine, Seoul, Korea
}

Rhabdomyoma is the most common fetal cardiac tumor, and its development is related to tuberous sclerosis. Fetal cardiac rhabdomyomas often spontaneously regress in utero or after birth, but large tumors can cause hemodynamic obstruction. Sirolimus, a mammalian target of rapamycin (mTOR) inhibitor, has been used as an immunosuppressant after organ transplantation. The mTOR inhibitors are well-known to have anti-tumor activity, and they have been used for the treatment of patients with tuberous sclerosis. In the current case, fetal cardiac rhabdomyoma was completely resolved in utero during oral sirolimus treatment in the mother with tuberous sclerosis. This case shows that oral sirolimus therapy in pregnancy may be a treatment for multiple or large fetal cardiac rhabdomyomas.

Keywords: Sirolimus; Cardiac tumor; Rhabdomyoma; Tuberous sclerosis; Fetal therapy

\section{Introduction}

Rhabdomyoma is the most common cardiac tumor in fetal life, accounting for $60-86 \%$ of primary fetal cardiac tumors [1]. They commonly regress spontaneously over time without treatment, but large tumors can cause hemodynamic obstruction and subsequent heart failure or arrhythmias and fetal hydrops. The prognosis depends on fetal cardiac complication in utero and is poor when there is fetal cardiac dysfunction [2]. Surgical resection is needed when the tumors cause severe ventricular inflow or outflow tract obstruction [3].

Sirolimus, an inhibitor of mammalian target of rapamycin (mTOR), has been used as an immunosuppressant in kidney and heart transplant recipients [3]. Recently, mTOR inhibitors have been used in patients with tuberous sclerosis. Mutations in either tuberous sclerosis complex (TSC1 or TSC2) causes abnormal activation of the mTOR pathway, leading to increased cell growth and proliferation. TSC1 or TSC2 dysfunction is responsible for the hamartomatous lesions of tuberous sclerosis [4].

Despite the known antitumor activity of mTOR inhibitors in adults, there have been few case reports of mTOR inhibitor therapy as a pharmacologic alternative to surgery demonstrating successful regression of cardiac rhabdomyoma in infants $[3,5]$. Moreover, there was only 1 case report that showed partial regression of obstructive fetal cardiac rhabdomyomas in utero via mTOR inhibitor treatment of the mother during pregnancy [1].

Here, we report a case of fetal cardiac rhabdomyomas that showed complete regression in utero via treatment with oral sirolimus of the mother.

\section{Case report}

A 32-year-old woman with gravida 2 visited the outpatient clinic at 19 weeks 5 days of pregnancy. Before pregnancy, she was diagnosed with tuberous sclerosis with pulmonary lymphangioleiomyomatosis (LAM) at the age of 24 . She had

Received: 2018.09.17. Revised: 2018.10.22. Accepted: 2018.10.26. Corresponding author: Cheong-Rae Roh

Department of Obstetrics and Gynecology, Samsung Medical Center, Sungkyunkwan University School of Medicine, 81 Irwonro, Gangnam-gu, Seoul 06351, Korea

E-mail: crroh@skku.edu

https://orcid.org/0000-0002-5266-6168

Articles published in Obstet Gynecol Sci are open-access, distributed under the terms of the Creative Commons Attribution Non-Commercial License (http://creativecommons. org/licenses/by-nc/3.0/) which permits unrestricted non-commercial use, distribution, and reproduction in any medium, provided the original work is properly cited.

Copyright $\odot 2019$ Korean Society of Obstetrics and Gynecology 


\section{Obstetrics \& Gynecology Science}

Hyea Park, et al. Sirolimus and fetal cardiac rhabdomyoma

pathologic genetic variant of c.5108dup (p.Ser1704Valfs*2) in TSC2. Since diagnosis, she was prescribed $1 \mathrm{mg}$ sirolimus daily to control pulmonary LAM, but she arbitrarily discontinued sirolimus after confirmation of the pregnancy. Genetic amniocentesis revealed the same TSC2 gene mutation in the fetus.

Fetal cardiac rhabdomyoma measuring $0.75 \times 0.57 \mathrm{~cm}$ in the right ventricle (RV) was first observed at 21 weeks 5 days of pregnancy during fetal echocardiography. After confirmation of drug discontinuation, oral sirolimus was re-started in the mother with target maternal trough serum level of $10-15 \mathrm{ng} / \mathrm{mL}$ since 23 weeks of pregnancy. The initial dose of sirolimus was $4 \mathrm{mg}$ per day.

Fetal echocardiogram at 24 weeks 5 days of pregnancy demonstrated multiple cardiac rhabdomyomas (1 tumor in the RV measuring $1.0 \times 1.0 \mathrm{~cm}$ and 2 tumors in the left ventricle [LV] measuring $1.2 \times 0.9 \mathrm{~cm}$ and $0.4 \times 0.3 \mathrm{~cm}$; Fig. $1 \mathrm{~A}$ and $\mathrm{B}$ ). The number and size of the fetal cardiac tumors increased despite oral $4 \mathrm{mg} /$ day sirolimus. The maternal serum level of sirolimus was $2.2 \mathrm{ng} / \mathrm{mL}$, and the dosage was increased to
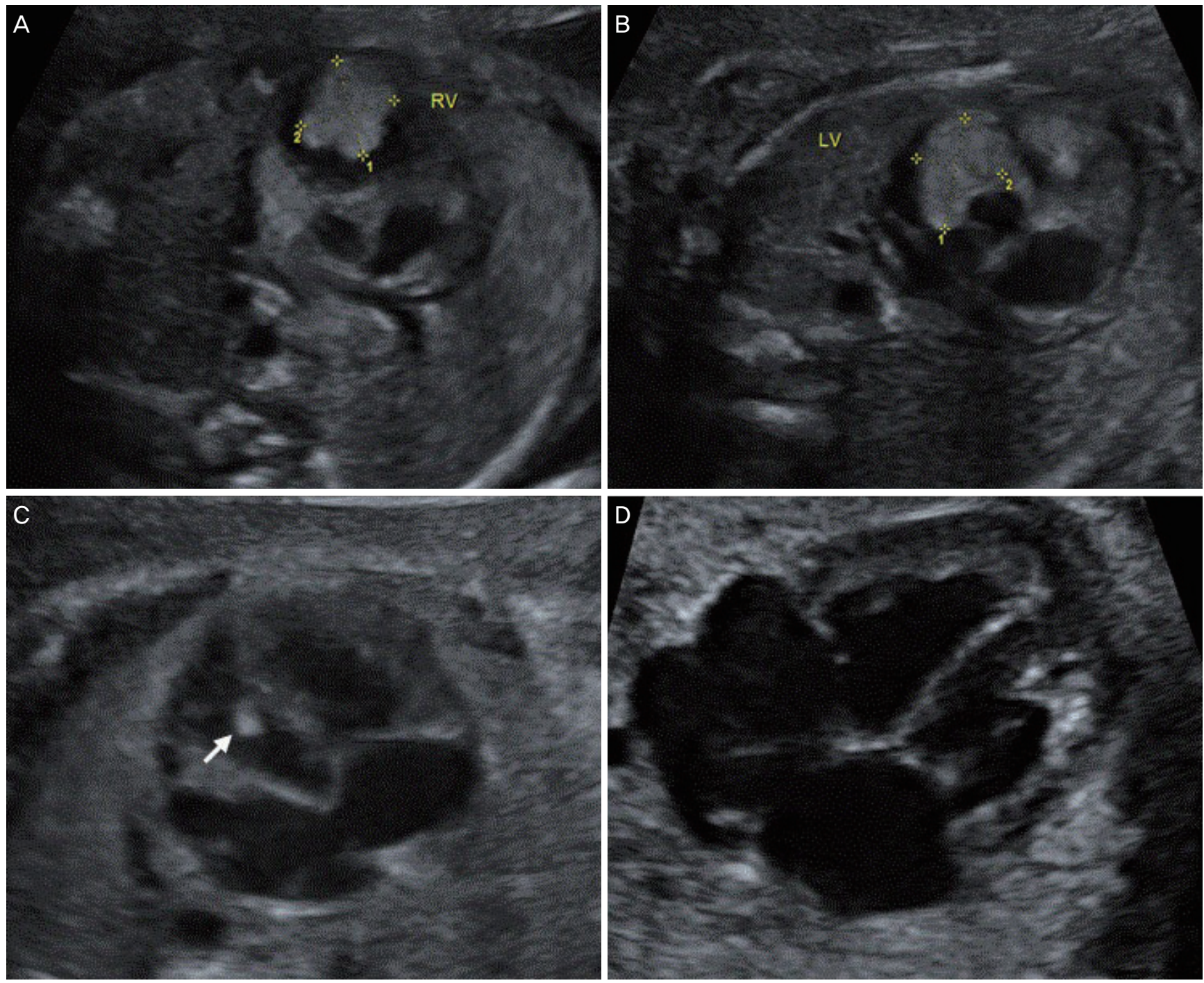

Fig. 1. Findings of fetal echocardiography. (A, B) Fatal echocardiogram of both ventricles at 24 weeks 5 days of pregnancy. Two large echogenic intracardiac masses were detected. $1.0 \times 1.0 \mathrm{~cm}$ sized tumor in RV, $1.2 \times 0.9 \mathrm{~cm}$ sized tumor in LV. (C) Fetal echocardiogram showing marked regression of fetal cardiac tumors at 27 weeks 5 days of pregnancy. Only one tiny cardiac tumor was detected on fetal echocardiography (size of $0.4 \times 0.2 \mathrm{~cm}$ in RV). (D) Fetal echocardiogram at 29 weeks 5 days of pregnancy showing no residual tumor in both $R V$ and $L V$. $R V$, right ventricle; $L V$, left ventricle. 


\section{Obstetrics \& Gynecology Science}

Vol. 62, No. 4, 2019

$12 \mathrm{mg} /$ day to achieve the planned therapeutic level.

Serial fetal echocardiography was performed to monitor the cardiac tumors. In 15 days after the sirolimus dosage was increased, maternal serum sirolimus level reached to therapeutic level $(12.1 \mathrm{ng} / \mathrm{mL})$, but the number and size of the cardiac tumors remained the same with those in the previous echocardiography (longest diameter measured $0.97 \mathrm{~cm}$ in RV and $1.24 \mathrm{~cm}$ and $0.82 \mathrm{~cm}$ in LV). However, after 19 days, the number and size of the tumors markedly decreased, and only 1 tiny cardiac tumor was detected on fetal echocardiography, which measured $0.4 \times 0.2 \mathrm{~cm}$ and was located in the RV (Fig. 1C).

Finally, at 29 weeks 5 days of pregnancy, no cardiac mass was observed on fetal echocardiography (Fig. 1D). Subsequent sonographic study also detected no cardiac mass or structural anomaly. However, $12 \mathrm{mg}$ of oral sirolimus daily was continued until delivery.

She delivered a male infant at 39 weeks of gestation. The infant weighed $3.22 \mathrm{~kg}$, and the 1 minute and 5 minutes Apgar scores were 9 and 10. At the time of delivery, the sirolimus level was $25.0 \mathrm{ng} / \mathrm{mL}$ in maternal serum and $33.2 \mathrm{ng} / \mathrm{mL}$ in the fetal cord blood. After delivery, the mother started to take sirolimus $1 \mathrm{mg}$ per day as before pregnancy. The infant did not receive any therapy for the high level of sirolimus in the fetal cord blood. One day after birth, echocardiography of the infant showed no intracardiac mass (Fig. 2A). However, brain sonography revealed multifocal cortical/subcortical tubers with suspicious subependymal nodules (Fig. 2B). Abdomen sonography also found suspicious echogenic lesion in both the kidney medullary areas (Fig. 2C). The infant is under follow-up on outpatient basis.

\section{Discussion}

Tuberous sclerosis is a genetic disease characterized by benign tumor lesions in multiple organs such as the brain, kidneys, heart, lungs, and skin. Two gene mutations have been identified to cause the disease, namely, TSC1 and TSC2 located on chromosome $9 q 34$ and 16p13.3, respectively. Both TSC1 and TSC2 are tumor suppressor genes, and the mutation of these genes lead to uncontrolled proliferation of benign tumors in various sites. This genetic disease is inherited in an autosomal dominant manner [6]. Tuberous sclerosis develops in approximately 1 per 6,000 to 10,000 population, and approximately $80 \%$ are caused by de novo mutation. However, its exact incidence rate is unknown because some patients are only mildly affected or even asymptomatic and are thus undiagnosed [7].

Patients with tuberous sclerosis present a variety of clinical manifestations. Common symptoms include skin lesions, seizures, learning disabilities, or manifestations of tumors affecting organs described above. Clinical attentions have been primarily paid to tumors involving the heart, brain, kidneys, and lungs. In general, tumor manifestations of tuberous sclerosis occur later in life, but cardiac rhabdomyomas are usually diagnosed early in life or prenatally. Majority of fetuses with multiple rhabdomyomas have tuberous sclerosis [6].

Fetal cardiac rhabdomyoma is usually identified in the midtrimester. Ultrasound scans of cardiac rhabdomyoma typically show the presence of echogenic masses localized intramurally. A familial history of tuberous sclerosis may be helpful in the diagnosis of rhabdomyoma as in our case [2]. The natural course of most tumors detected prenatally is favorable,
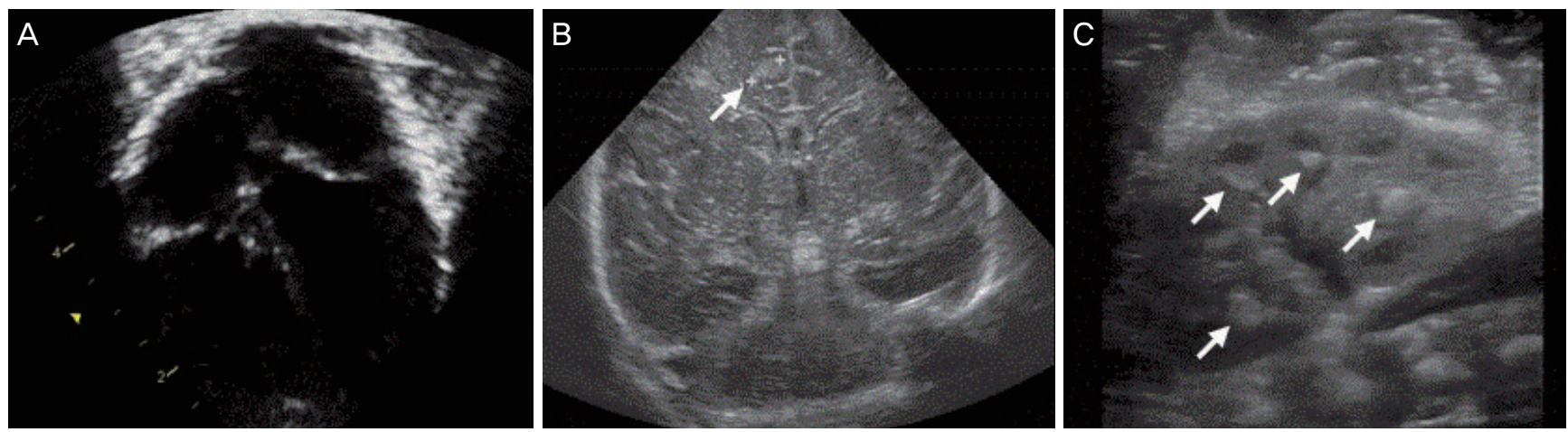

Fig. 2. Findings of neonatal sonography. (A) Neonatal echocardiography showed no intracardiac tumor 1 day after birth. (B) Brain sonography of neonate revealed multifocal subcortical tubers suspicious subependymal nodules. (C) Abdomen sonography also found suspicious echogenic lesions in both kidney medullary area. 


\section{Obstetrics \& Gynecology Science}

Hyea Park, et al. Sirolimus and fetal cardiac rhabdomyoma

showing tumor regression; however, they may also progress in utero. After birth, large tumors may affect the cardiac output; in such cases, surgical resection or further treatment will be needed [7]. Close follow-up with fetal echocardiography may be helpful for determining the appropriate timing of delivery to avoid significant hemodynamic complications in these cases.

Sirolimus is a first-generation inhibitor of mTOR protein kinase that controls cell growth, proliferation, and survival. Initially, mTOR inhibitor was prescribed for its anti-cancer activity, but it has also been approved by the Food and Drug Administration of the Unites states as an immunosuppressant after organ transplantation. It prevents activation of $T$ cells and B cells by inhibiting their response to interleukin-2. The most commonly reported adverse effects of sirolimus are hypertriglyceridemia, hypercholesterolemia, peripheral edema, hypertension, and creatinine increase [6].

The mechanism by which placental transfer of sirolimus occurs is unknown. The molecular weight of sirolimus (914 Da) is within the range for passive diffusion, and its elimination half-life of 46 to 78 hours is enough to allow the drug to be present at the maternal-fetal interface. However, the high $92 \%$ plasma protein binding may impede and limit the amount of drug available for placental transfer $[1,8,9]$. In the current case, the cord blood level of sirolimus at birth was $132 \%$ of that in maternal serum, suggesting that sirolimus crosses the placenta, and the transfer is preferentially unidirectional. As the maternal serum sirolimus level reached to therapeutic target level, the fetal cardiac tumors also rapidly regressed, showing a very strong timing correlation.

The fetal effects of mTOR inhibitors are still poorly defined because limited data are available, but these immunosuppressive drugs do not seem to be an absolute contraindication in pregnancy. However, we need to consider the potential effects these drugs may have in pregnancy, including hyperlipidemia and diabetogenic effects. In cases in which the mothers are at high risk for gestational diabetes, worsening lipid and glucose metabolism should be avoided. Earlier screening should also be considered [10].

In the current case, the dose of sirolimus used in the mother was based on previous cases of rhabdomyoma treated with sirolimus therapy $[1,3]$. In the previous cases, the target serum sirolimus level was decided based on the guidelines for sirolimus use in adult transplant patients. Commonly, the target sirolimus levels in transplant recipients range from
$10 \mathrm{ng} / \mathrm{mL}$ to $15 \mathrm{ng} / \mathrm{mL}$ [11]. Meanwhile, further studies are needed to determine the safety and efficacy of sirolimus therapy in pregnant women. Further evaluation is also needed to determine the most appropriate dose and therapeutic maternal serum level of sirolimus for the treatment of fetal cardiac rhabdomyomas.

This report demonstrates that sirolimus may be helpful for the regression of fetal cardiac rhabdomyomas. Although there is 1 previous report of sirolimus therapy in the mother whose fetus had cardiac rhabdomyomas, complete resolution of cardiac rhabdomyoma has not been previously reported [1]. In pregnant women with tuberous sclerosis, fetal targeted sonography is mandatory. Once diagnosis of rhabdomyoma is established, continued surveillance is necessary to monitor the progression of known lesions and the emergence of new ones. If multiple cardiac rhabdomyomas increase in size, sirolimus therapy has the potential to induce regression of rhabdomyomas in the fetal heart.

\section{Conflict of interest}

No potential conflict of interest relevant to this article was reported.

\section{Ethical approval}

The study was approved by the Institutional Review Board of Samsung Medical Center (IRB No. 2019-06-070) and performed in accordance with the principles of the Declaration of Helsinki.

\section{References}

1. Barnes BT, Procaccini D, Crino J, Blakemore $K$, Sekar $P$, Sagaser KG, et al. Maternal sirolimus therapy for fetal cardiac rhabdomyomas. N Engl J Med 2018;378:18445.

2. Fesslova V, Villa L, Rizzuti T, Mastrangelo M, Mosca F. Natural history and long-term outcome of cardiac rhabdomyomas detected prenatally. Prenat Diagn 2004;24:241-8.

3. Breathnach C, Pears J, Franklin O, Webb D, McMa- 


\section{Obstetrics \& Gynecology Science}

Vol. 62, No. 4, 2019

hon CJ. Rapid regression of left ventricular outflow tract rhabdomyoma after sirolimus therapy. Pediatrics 2014;134:e1199-202.

4. Ninic S, Kalaba M, Jovicic B, Vukomanovic V, Prijic $S$, Vucetic $B$, et al. Successful use of sirolimus for refractory atrial ectopic tachycardia in a child with cardiac rhabdomyoma. Ann Noninvasive Electrocardiol 2017;22:e12435.

5. Demir HA, Ekici F, Yazal Erdem A, Emir S, Tunç B. Everolimus: a challenging drug in the treatment of multifocal inoperable cardiac rhabdomyoma. Pediatrics 2012;130:e243-7.

6. Sasongko TH, Ismail NF, Zabidi-Hussin Z. Rapamycin and rapalogs for tuberous sclerosis complex. Cochrane Database Syst Rev 2016;7:CD011272.

7. Bader RS, Chitayat D, Kelly E, Ryan G, Smallhorn JF, Toi A, et al. Fetal rhabdomyoma: prenatal diagnosis, clinical outcome, and incidence of associated tuberous sclerosis complex. J Pediatr 2003;143:620-4.

8. Rapamune (sirolimus) oral solution and tablets. Philadelphia (PA): Wyeth Pharmaceuticals, Inc; 2012.

9. Briggs $G G$, Freeman RK, Towers CV, Forinash AB. Drugs in pregnancy and lactation: a reference guide to fetal and neonatal risk. 10th ed. Philadelphia (PA): Wolters Kluwer; 2015.

10. Framarino-dei-Malatesta $M$, Derme $M$, Manzia TM, laria G, De Luca L, Fazzolari L, et al. Impact of mTOR-I on fertility and pregnancy: state of the art and review of the literature. Expert Rev Clin Immunol 2013;9:781-9.

11. Costanzo MR, Dipchand A, Starling R, Anderson A, Chan M, Desai $S$, et al. The International Society of Heart and Lung Transplantation Guidelines for the care of heart transplant recipients. J Heart Lung Transplant 2010;29:914-56. 